\title{
Ovarian Germ Cell Tumours: Clinicopathological Features and Treatment Outcomes
}

\author{
N.Naqos, A.Taleb, Z.Bouchbika, N.Benchakroun, H.Jouhadi, \\ N.Tawfiq, S.Sahraoui, A.Benider Mohammed \\ VI Center Of Cancer Treatment, Casablanca, Morocco.
}

\begin{abstract}
Malignant germ cell ovarian tumors (MOGCTs) represent less than $20 \%$ of ovarian cancers. We conducted a retrospective study of 16 cases of (MOGCTs) diagnosed from January 2008 to December 2013. Results: During this time 26 cases of non epithelial ovarian malignancy were diagnosed which 16 are germinal tumors $61 \%$. The age of patients ranged from 16 to 60 years. Abdominal mass was the principal symptom, it was present in 2/3 of cases (66\%). All patients were surgically assessed with ovariectomy that was associated with hysterectomy in two patients. Chemotherapy was administered in 15 patients and BEP was the commonest regimen used. After a mean follow up of 25 months we obtained a clinical, biological and radiologic complete remission in 1/3 of cases, 3 recurrences, 2 deaths.

Overall survival: After a median follow up of 25 months. We noted 2 death. The median OS was not reached. The 2-year OS rate was 93.8\%. Event free survival (EFS): Events were defined as death, recurrence or progression of the disease. The median EFS was not reached.The 2-year EFS rate was $73 \%$.

Conclusion: (MOGCTs)are rare; they generally affect the young woman and are diagnosed at an early stage. Surgery has an important role.
\end{abstract}

Keywords: germ cells, ovarian cancer, non epithelial.

\section{Introduction}

Non-epithelial ovarian malignancies account for about $10 \%$ of all ovarian cancers. Malignant ovarian germ cell tumors (MOGCTs) represent 5\% of all ovarian cancers and are mostly diagnosed in young women [1] .It can be subdivided into dysgerminoma, endodermal sinus tumor (EST), emlbryonal carcinoma, polyembryoma, choriocarcinoma, immature teratoma (Imm T), and mixed germ cell tumor (MGCT). MOGCTs are rare but aggressive and very curable tumors at all presenting stages of disease, multimodality therapy with initial fertility-sparing surgery and platinum-based chemotherapy generally is regarded as the standard of care and has improved the prognosis significantly. Unlike epithelial ovarian cancer (EOC), MOGCTs grow rapidly. Experience with MOGCTs is much more limited than testicular tumors, with little available randomized data, furthermore Little is known about ovarian GCTs in Morocco. Thus, we conducted this study to report the clinico-pathological features, treatments and outcomes of ovarian GCTs at a big Moroccan Cancer Center.

\section{Materiels and methods}

This is a retrospective analysis of all cases of malignant ovarian germ cell tumors pathologically proven and diagnosed between Junuary 2007 and December 2012, Data from medical records were collected and analyzed including age of diagnosis, primary surgery, histologic type of tumor, initial stage and grade of the cancer, treatments and their outcomes.

\section{Results}

During this period 26 cases of non epithelial ovarian cancer were unregistered from witch 16 germ cell tumors $61 \%$ Age ranged between 16 and 60 years with a median of 40 years. Almost $90 \%$ of patients were below the age of 40 years (Table 1 ).

Ten patients were nulliparous (62\%), two cases of oral contraception, (16,6\%) 6 patients are menopausal (37\%), 3 had a familiar history of breast cancer. The mean duration of consultation is 6 months [2-24 months]. Concerning symptoms, abdominal mass was the principal consultation cause, it was present in $2 / 3$ of cases (66\%). $62 \%$ of patients had pelvic ache, 3 had amenorrhea whereas 6 had metrorragy, one case of virilisation was noted.

All patients were surgically assessed with ovariectomy that was associated with hysterectomy in two patients with the diagnosis of malignant teratoma and stage IIIC. One patient was 68 years old and postmenopausal and thus fertility preservation was not applicable. The other patient was 35 years and the diagnosis of GCTs was done postoperatively. Complete surgical resection (R0) was the rule. 
Chemotherapy was administered in 15 patients (\%) and BEP was the commonest regimen that was used in 14 cases, and etoposide- platine in one case. The median number of cycles was 4 (range, 1-6). Beyond 4 cycles, bleomycin was omitted. Toxicity was documented only in 3 patients and was mostly hematologic (neutropenia). None has documented pulmonary toxicity.

After a mean follow up of 25 months we noticed a clinical, biological and radiologic complete remission in 1/3 of cases (37,5\%), 3 recurrences , 2 patients died. Overall survival: After a median follow up of 25 months. We noted 2 death. The median OS was not reached (Fig. 1). The 2-year OS rate was 93.8\%. Event free survival (EFS): Events were defined as death, recurrence or progression of the disease. The median EFS was not reached (Fig. 2). The 2- year EFS rate was 73\%.

Discussion:

Malignant ovarian germ cell tumors (MOGCTs) consist of a broad spectrum of tumor types histogenetically derived from primordial germ cells and sex-stromal derivatives that differ with regard to clinical presentation, tumor biology, and histology. MOGCTs are rare but aggressive and very curable tumors at all presenting stages of disease, accounting for approximately $1-2 \%$ of all ovarian malignancies [1] In our study, the median age was 23 years (range 18-68 years), which was higher than 19 years reported by Talukdar et al. [2]. In the first 2 decades of life, almost $70 \%$ of ovarian tumours are of germ-cell origin, and one-third of these are malignant [3].

Symptoms and signs of MOGCTs are rather typical. MOGCTs tend to be quite large and rapidly progressive malignancies [4,5], Abdominal pain associated with a palpable pelvic- abdominal mass is observed in about $85 \%$ of patients. Fever is present in $10-25 \%$ of the cases. Ascites or peritonitis secondary to torsion, infection, or rupture of the ovarian tumor, is other possible clinical features [6].

Histologically MOGCT are divided into dysgerminoma (the most common type) and nondysgerminomatous tumors. The most common types of non dysgerminomatous tumors are yolk sac tumors, immature teratoma, and mixed germ cell tumors. Less common variants of non-dysgerminomatous tumors are embryonal carcinoma, polyembryoma, and non gestational choriocarcinoma.

Teratomas were the most common pathologies In this study. European data shows those teratomas are the most common GCTs [8].

In the current study, fertility preservation surgery was achieved in 16 patients. However, two patients had hysterectomy with bilateral annexectomy. One was in the postmenopausal period (68 years) while the other was 35 years of age and both were stage IIIC, with teratoma histology. This is similar to the published guidelines where unilateral salpingo-oophorectomy with preservation of the contralateral ovary and the uterus is considered the adequate surgical treatment for patients with GCTs $[9,10]$. In women with advanced disease ( para-aortic lymph-node metastases), preservation of reproductive function is also possible, particularly if the contralateral ovary is normal $[11,12]$ patients with stage Is/grade 1 immature teratoma can be safely treated with unilateral oophorectomy, and no more chemotherapy is required. Stage Ia grade 2 or 3 as well as stage $\mathrm{Ib}$ through IIc disease require short-term chemotherapy for three to six courses. Long-term chemotherapy,perhaps 1 year with combination therapy, might be more effective for advanced-stage immature teratoma than the short-term therapy [13]. After the report in 1977 of the activity of the combination of cisplatin, vinblastine and bleomycin (PVB) in testicular cancer, platinum-based chemotherapy became the standard regimen for patients with MOGCTs [14]. Subsequent developments in testicular cancer defined the activity of etoposide and later this drug was incorporated into the combination regimens. Thus, BEP chemotherapy is regarded to be the gold-standard regimen for the first-line treatment of germ cell tumors at all stages of disease [14]. Several studies have been accomplished on EP with the aim of a less toxic regime. An EORTC study indicated that no differences were found between BEP and EP in good-prognosis patients, so EP can be regarded as the standard treatment for such patients [15].

In the current study, the median OS was not reached reflecting the good prognosis. Patients responding to chemotherapy have significantly better 5 -year OS rates (100\%) than non- responders $(0 \%)$.

The experience by the GOG [16], noted that completely resected patients (R0) had better OS than those who had residual after surgery. Williams et al. [17] found out that patients with no residual after surgery had significantly better EFS than those with gross residual.

\section{Conclusion}

MOGCTs are relatively uncommon neoplasms that are characterized by high chemosensitivity. Surgery has an important role in the management of MOGCTs patients. Initial careful surgical staging is of great importance for selection of appropriate subsequent therapy. Fertility-sparing surgery is feasible in most cases with early stage disease.

Finally, we are in need of multicenter and multinational prospective analysis of treatment option for this disease over several years with a large number of patients so as to understand their successes and failures. 


\section{References}

[1]. Quirk JT, Natarajan N, Mettlin CJ. Age-specific ovarian cancer incidence rate patterns in the United States. Gynecol Oncol 2005;99(1):248-50.

[2]. Talukdar S, Kumar S, Bhatla N, Mathur S, Thulkar S, Kumar L. Neo-adjuvant chemotherapy in the treatment of advanced malignant germ cell tumors of ovary. Gynecol Oncol 2013. http://dx.doi.org/10.1016/j.ygyno.2013.10.009, pii: S0090- 8258(13)01254-7.

[3]. Chen LM, Berek JS. Ovarian and fallopian tubes. In: Haskell CM, editor. Cancer treatment. Philadelphia: WB Saunders; 2000. p. 900-32.

[4]. Norris HJ, Zirkin HJ, Benson WL. Immature (malignant) teratoma of the ovary: a clinical and pathologic study of 58 cases. Cancer 1976;37(5):2359-72.

[5]. Gershenson DM, del Junco G, Silva EG, Copeland LJ, Wharton FN, Rutledge FN. Immature teratoma of the ovary. Obstet Gynecol 1986;68(5):624-9.

[6]. Tewari K, Cappuccini F, Disaia PJ, Berman ML, Manetta A, Kohler MF. Malignant germ cell tumors of the ovary. Obstet Gynecol 2000;95(1):128-33.

[7]. Mokhtar N, Gouda I, Adel I. Cancer pathology registry 2003-2004 and time trend analysis. Egypt: Department of Pathology, NCI; 2007.

[8]. Colombo N, Peiretti M, Garbi A, Carinelli S, Marini C, Sessa C, on behalf of the ESMO Guidelines Working Group. Nonepithelial ovarian cancer: ESMO clinical practice guidelines for diagnosis, treatment and follow-up. Ann Oncol 2012;23(Suppl. 7):vii20-6.

[9]. Gershenson DM. Management of ovarian germ cell tumors. J Clin Oncol 2007;25:2938-43.

[10]. Pectasides D, Pectasides E, Kassanos D. Germ cell tumors of the ovary. Cancer Treat Rev 2008;34:427-41.

[11]. Low JJ, Perrin LC, Crandon AJ, Hacker NF. Conservative surgery to preserve ovarian function in patients with malignant

[12]. ovarian germ cell tumors. A review of 74 cases. Cancer 2000;89:391-8.

[13]. Colombo N, Parma G, Zanagnolo V, Insinga A. Management of ovarian stromal cell tumors. J Clin Oncol 2007;25:2944-51.

[14]. S.-N. Chow*a9b, J.-H. Yanga Malignant ovarian germ cell tumors International Journal of Gynecology \& Obstetrics 53 (1996) $151-158$

[15]. D. Pectasides a,*, E. Pectasides Germ cell tumors of the ovary Cancer Treatment Reviews $(2008) 34,427-441$

[16]. V. Guillem and A. Poveda Germ cell tumours of the ovary Clin Transl Oncol (2007) 9:237-243

[17]. DOI 10.1007/s12094-007-0045-0

[18]. Einhorn LH, Williams SD, Chamness A, Brames MJ, Perkins SM, Abonour R. High-dose chemotherapy and stem-cell rescue for metastatic germ-cell tumors. N Engl J Med 2007;357(4):340-8.

[19]. Williams S, Blessing JA, Liao SY, Ball H, Hanjani P, et al. Adjuvant therapy of ovarian germ cell tumors with cisplatin, etoposide, and bleomycin: a trial of the Gynecologic Oncology Group. J Clin Oncol 1994;12:701. No conflit of interest.

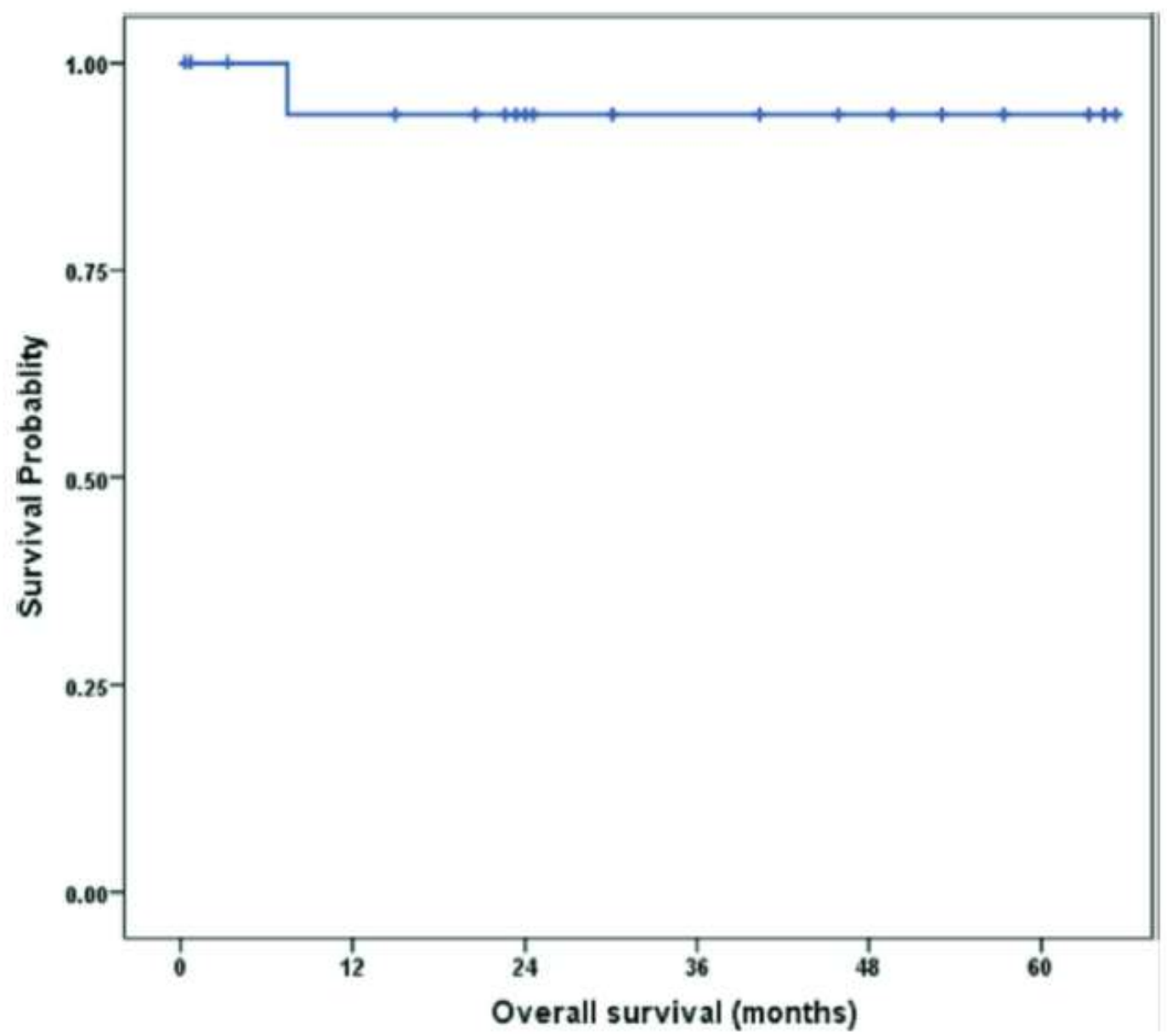

Figure 1 OS of female patients with GCTs. 


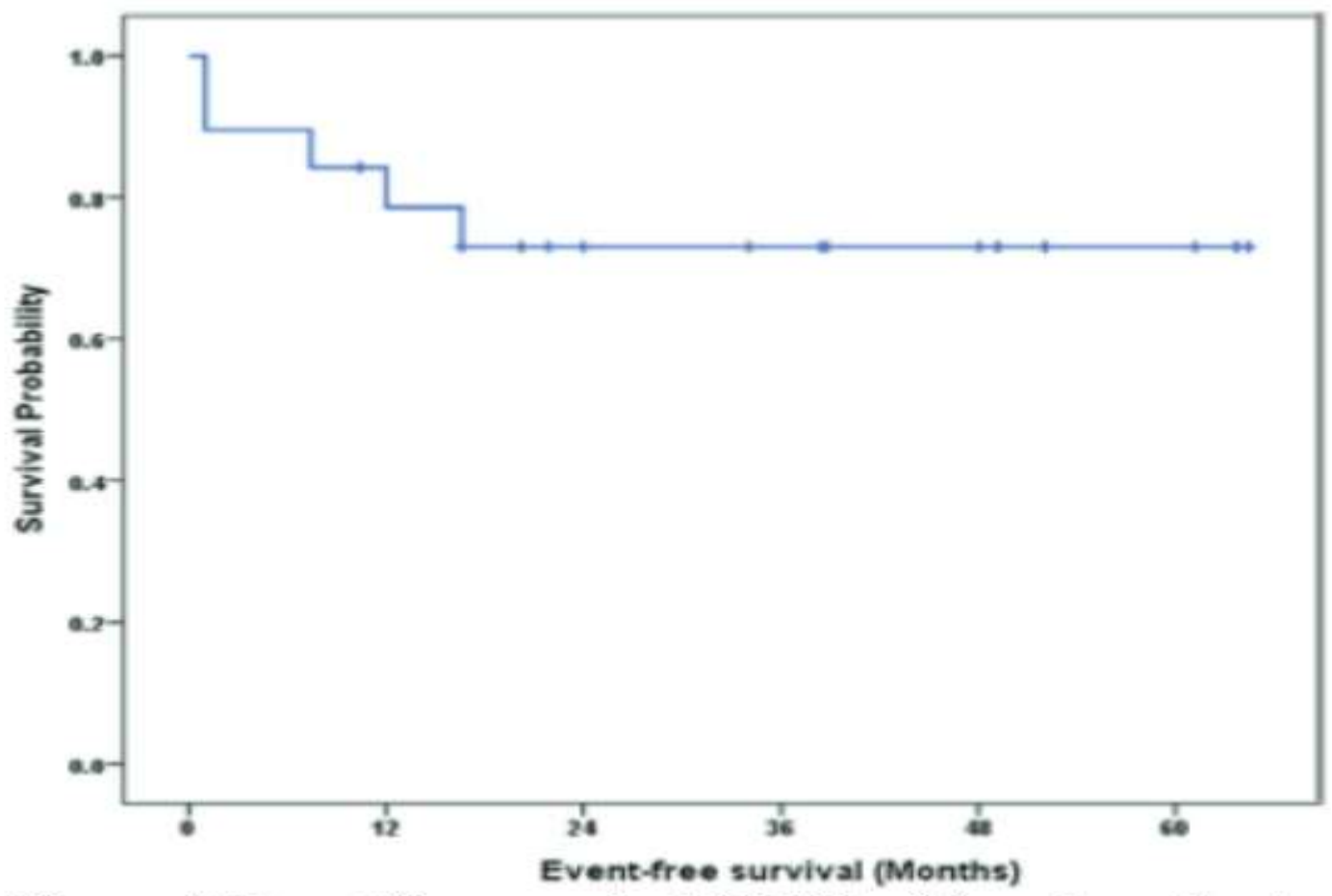

Figure 2 Event free survival (EFS) of female patients with GCTs.

TABLE 1: Characterisctics of patients with germ cell tumors:

\begin{tabular}{|c|c|c|}
\hline \multicolumn{2}{|c|}{$\begin{array}{l}\text { Characteristics of female patients with germ cell } \\
\text { tumors }\end{array}$} & N.(\%) \\
\hline \multicolumn{2}{|l|}{ total } & 16 \\
\hline \multicolumn{2}{|c|}{ Median age (range years) } & 25 years old ( $18-68)$ \\
\hline \multicolumn{2}{|c|}{ Median Time to diagnosis } & 2 months (2-24 months) \\
\hline \multirow[t]{5}{*}{ Presentation } & Swelling & \\
\hline & |pain & $\mid 10(62 \%)$ \\
\hline & Abdominal mass & $12(75 \%)$ \\
\hline & metrorragy & $6(37.5 \%)$ \\
\hline & amenorrhee & $3(19 \%)$ \\
\hline \multirow[t]{4}{*}{ Pathology } & dysgerminoma & 0 \\
\hline & Teratoma & $12(75 \%)$ \\
\hline & |Vole sac tumor & $2(12,5 \%)$ \\
\hline & Embryonnar carcinoma & $2(12,5 \%)$ \\
\hline \multicolumn{3}{|l|}{ FIGO stage } \\
\hline I & & $2(12,5 \%)$ \\
\hline |II & & 0 \\
\hline III & & $4(25 \%)$ \\
\hline IIV & & $6(37.5 \%)$ \\
\hline
\end{tabular}

